\author{
ARTICLES \\ MARZENA BLACHOWSKA-SZMIGIEL \\ majek@amu.edu.pl \\ Université de Poznań
}

\title{
LE COACHING TROUVERA-T-IL SA PLACE DANS LE DÉVELOP- PEMENT DES COMPÉTENCES CRÉATIVES DES ENSEIGNANTS DU FLE EN POLOGNE?
}

\begin{abstract}
Marzena Blachowska-Szmigiel, Le coaching trouvera-t-il sa place dans le développement des compétences créatives des enseignants du FLE en Pologne? [Can coaching techniques be successfully implemented in working up creative skills of Polish FFL teachers?], Studia Romanica Posnaniensia, Adam Mickiewicz University Press, Poznań, vol. XLI/3: 2014, pp. 3-15. ISBN 978-83-232-2759-5. ISSN 0137-2475. eISSN 2084-4158. DOI: 10.7169/strop2014.413.001

The teaching profession, including the language teaching profession, requires that teachers should constantly enhance their professional competences. Among those competences, the creative ones occupy a fixed position. In this paper, I am going to consider the possibility of optimizing their development by the use of coaching. In spite of controversies aroused by this work method, it can offer a significant support in developing the creative competences of French language teachers.
\end{abstract}

Keywords: coaching, creative competences, French language teaching profession

Une fois ses études graduantes finies, l'enseignant du FLE les complète par les stages, les ateliers, les études postgraduantes, etc. C'est ainsi qu'il construit son autorité et sa carrière professionnelle. Dans le présent article je réfléchis sur les possibilités d'optimiser le développement des compétences créatives de l'enseignant du FLE par le coaching entendu comme une forme d'accompagnement. L'article est divisé en trois parties : dans la première partie j'aborde la question de l'évolution du rôle de l'enseignant du FLE, dans la partie suivante je cherche à montrer la croissance de l'intérêt pour les compétences créatives de l'enseignant (y compris de l'enseignant du FLE), dans la dernière partie j'examine les intérêts et les défis du coaching en tant qu'outil d'optimisation du développement de la créativité des enseignants du FLE. 


\section{L'ENSEIGNANT DU FLE : MAÎTRE, GUIDE OU COACH ?}

Dans le paradigme de l'enseignement transmissif, dominant en didactique des langues étrangères (LE) dans la première moitié du $\mathrm{XX}^{\mathrm{ème}}$ siècle, l'enseignant était une infaillible source de savoir langagier. Ses relations avec l'élève s'appuyaient sur des règles précises, nettes et transparentes dont le fondement constituait le style autoritaire de la gestion du processus didactique et éducatif. À l'époque, la fonction didactique de l'enseignant des LE consistait à transmettre des règles de grammaire, à développer les habitudes de leur utilisation et à évaluer leur maîtrise. Quant à l'élève, comparé souvent à « une boîte vide », il devait maîtriser les règles et, grâce à un nombre suffisant de répétions, les utiliser correctement. La fonction éducative de l'enseignant se limitait à maintenir la discipline en classe. Dans ce modèle béhavioral de l'enseignement, dépourvu d'individualisme et de nonconformisme, l'autorité de l'enseignant reposant sur son statut formel, était incontestable. L'enseignant ordonnait et interdisait, l'élève écoutait et répétait sans trop poser de questions.

À partir de la deuxième moitié du $\mathrm{XX}^{\text {ème }}$ siècle, la didactique des LE entre dans une période de changements dynamiques, laquelle conduit à la naissance du paradigme de l'enseignement « activisant » avec deux approches les plus représentatives, à savoir l'approche communicative et l'approche actionnelle. Il est à souligner que la naissance du paradigme activisant est considérablement influencée par les phénomènes en psychologie et en linguistique suivants :

- les conceptions cognitive et humaniste de l'homme selon lesquelles l'individu est intradirigé, autonome, capable de se poser des objectifs et de viser à les atteindre ;

- une vue cognitive et constructiviste de l'apprentissage selon laquelle l'apprentissage est une activité processuelle de la construction/la croissance du savoir et des savoir-faire ;

- l'évolution des conceptions de la langue des approches structuralistes par les approches générativistes jusqu'aux approches communicativistes, autrement dit de la langue en tant que système de structures lesquelles s'imbriquent les unes dans les autres jusqu'aux conceptions de la langue comme un ensemble aux frontières indéterminables dont la fonction est de créer des contenus imprévus par les systèmes.

Avec les approches communicative et actionnelle le « magistrocentrisme » cède la place au «puérocentrisme » (Meirieu, 2001). L'apprenant gagne donc le droit à commettre des fautes, à poser des questions, et même à évaluer l'efficacité des méthodes de travail de l'enseignant. Il est « acteur autonome de son apprentissage » (Martinez, 1996 : 76), et le développement de son autonomie devient l'un des défis principaux de la didactique des LE des années 90. du XX ${ }^{\text {ème }}$ siècle (Wilczyńska, 1999 ; Bogdański, 2001). Quant à l'enseignant, il est considéré comme animateur, intermédiaire, conseiller, facilitateur, guide, en bref une personne dirigeant le processus d'apprentissage avec une participation active de l'apprenant. Pourtant, si encore dans l'approche communicative l'enseignant veille sur les étapes de réalisation des activités et des 
exercices, dans l'approche actionnelle c'est l'apprenant-acteur social, qui le fait en coopérant avec d'autres apprenants-acteurs sociaux pendant la réalisation des projets. De son côté, l'enseignant-acteur social doit être en mesure de fournir des rétroactions à ses apprenants et de tenir compte de leurs rétroactions. Leur communication repose sur l'échange et, contrairement à l'époque du « magistrocentrisme », elle est de nature bidirectionnelle.

Un manager ou bien un coach ? Dans quelle direction évolue le rôle de l'enseignant de FLE d'aujourd'hui ? Ce qui est sûr c'est que son autorité n'est plus fondée sur son statut formel comme c'était à l'époque « magistrocentrique » mais sur ses dispositions, ses savoirs et ses savoir-faire, en bref sur ses compétences (Strykowski, 2005 : 16-17) qui vont bien au-delà des savoirs et savoir-faire méthodologiques (voir 2). D'une part l'enseignant de FLE d'aujourd'hui remplit toutes les fonctions d'un directeur : il planifie, organise, motive, évalue et résout des conflits. D'autre part, l'enseignant doit être en mesure de préparer les sujets apprenants à se retrouver dans la société contemporaine (voir par exemple Zawadzka, 2004 : 147) postmoderniste, globalisante et postindustrielle en pleine transition du paradigme de valeurs. Il doit également savoir les accompagner dans le développement de leurs compétences, non seulement langagières mais aussi générales, reposant sur les savoirs, les aptitudes et les savoir-faire, les savoir-être et les savoir-apprendre (CERCL, 2001).

Ainsi, l'enseignement du FLE semble s'approcher de plus en plus du coaching, entendu entre autres comme «l'accompagnement de personnes ou d'équipes pour le développement de leurs potentiels et de leurs savoir-faire dans le cadre d'objectifs professionnels » (Fatien, 2008). Pareillement à un coach qui guide et soutient son client, l'enseignant du FLE d'aujourd'hui guide et soutient le développement de l'apprenant, et ses objectifs principaux consistent à l'aider :

- à créer des relations de coopération avec les autres sujets apprenants en classe de langue ; ainsi qu'avec son entourage plus éloigné ;

- à mieux utiliser ses ressources, ses capacités et ses dispositions ;

- à élaborer des stratégies d'apprentissage efficaces.

Malgré ces ressemblances de fond, les différences entre ces deux métiers restent tranchantes. Premièrement, il serait difficile de comparer le statut social d'un enseignant et celui d'un coach. Contrairement au métier de coach qui devient en Pologne de plus en plus prestigieux, voire recherché, le métier d'enseignant de FLE est toujours sous-estimé. Deuxièmement, le coaché est intéressé par le développement de ses compétences pour des raisons professionnelles et/ou privées, ce qui n'est pas toujours le cas de l'apprenant en FLE surtout au collège ou au lycée.

La croissance de la complexité des fonctions et du rôle de l'enseignant moderne du FLE éveille des questions sur la nature et les types de compétences qu'il devrait avoir pour faire face à toutes ces exigences, ainsi que sur les façons de former les futurs enseignants et de développer les compétences des enseignants. Nous les abordons dans la suite du présent article. 


\section{LA CRÉATIVITÉ ET L’INNOVATION EN TANT QUE COMPÉTENCES FONDAMENTALES DE L'ENSEIGNANT DES LE D'AUJOURD'HUI}

La dernière décennie du $\mathrm{XX}^{\text {ème }}$ siècle est en Europe une période de réformes intenses de l'instruction publique accompagnée de discussions sur les compétences des enseignants (Dereń, 2011). En Pologne, les critiques de l'école, du système éducatif et du système de la formation des enseignants sont violentes. L'école, comme lieu d'éducation, est critiquée, entre autres, pour sa résistance aux innovations, sa tendance à uniformiser et à freiner la pensée ainsi que pour sa transmission du « savoir stérile » et pour l'affaiblissement de sa fonction didactique et éducative (Nęcka, 2005 : 149). L'école, comme lieu de travail, est critiquée avant tout pour sa bureaucratie, sa gestion centralisée et ses conditions du travail déplorables. Vu de la perspective des ressources hu-maines et du développement des organisations, l'école est loin de mettre en pratique les principes de l'organisation apprenante. En vogue depuis les années 90 . du $\mathrm{XX}^{\text {ème }}$ siècle, cette conception s'appuie sur l'idée de P. Senge (1998) selon laquelle l'organisation devrait gérer l'apprentissage de ses membres. Autrement dit, elle devrait être un lieu où les gens dirigés par le besoin de réalisation, améliorent constamment leurs capacités, apprennent comment apprendre ensemble et découvrent qu'ils sont constructeurs de la réalité.

Quant au système éducatif, il est critiqué pour l'inertie de ses actions et le manque de coordination de ses réformes avec le système de formation des enseignants. Les chercheurs soulignent la divergence croissante entre les exigences de l'éducation contemporaine et les possibilités d'y faire face par les enseignants ainsi que la nécessité d'une réforme de l'éducation des enseignants (Dereń, 2011 ; Zawadzka, 2004 : 52-62). Ils remarquent que la formation des enseignants devrait intégrer les savoirs et les savoir-faire, développer les attitudes et la conscience « d'être enseignant» (Dereń, 2011) et qu' « il est nécessaire d'intégrer la pédagogie, la psychologie et la méthodologie » (Zawadzka, 2004 : 55). Il faudrait que l'enseignant soit préparé à remettre en question son savoir et à prendre la responsabilité de ses actions (Dereń, 2011). De plus, vu son rôle de guide/de coach plutôt que de maître, il s'avère indispensable que les compétences de l'enseignant moderne soient plutôt ouvertes et créatives (Kwieciński, 2000).

Tout au long de la discussion sur les compétences de l'enseignant, les chercheurs en Pologne en proposent des classements divers. En voici les exemples les plus éminents des deux dernières décennies, mis en ordre chronologique :

- pour Z. Żukowska (1993 : 7-12), l'enseignant devrait se caractériser par les compétences substantielles-méthodologiques, méthodologiques-organisationnelles et socio-culturelles ;

- M. Taraszkiewicz (2001 : 175) décrit les compétences substantielles, didactiques-méthodologiques et éducatives ;

- E. Zawadzka (2004 : 110-111) énumère les compétences de communication, psycho-pédagogiques, méthodologiques, éducatives, organisationnelles, innovatrices et créatrices, de média ; 
- E.I. Laska (2007 : 108-115) parle des compétences substantielles, méthodologiques et organisationnelles et souligne la nécessité de leur constante actualisation ;

- E. Bilińska-Suchanek et M. Jarecka-Żyluk (2005 : 60) soulignent l'importance des compétences cognitives, critiques, interpersonnelles et décisives ;

- W. Strykowski (2005 : 18-27) caractérise les compétences substantielles, méthodologiques, psycho-pédagogiques, diagnostiques, de planification et de projet, didactiques, communicatives, de média, d'évaluation des programmes et des manuels, autoéducatives ;

- J. Jaroszewska (2009 : 85-90) complète le classement de W. Strykowski par les compétences éducatives, morales, techniques, innovatrices et créatrices.

En ce qui concerne les compétences de l'enseignant des LE, Zawadzka (2004: 110-111) complète son classement mentionné ci-dessus par les compétences langagière et culturelle, et Jaroszewska (2009 : 90) par la compétence interculturelle. Ce qui frappe dans ces classements exemplaires, c'est que leurs auteurs se soucient de fournir des répertoires des compétences sans vraiment se poser les questions de savoir comment les modéliser voire les opérationnaliser. Un bon exemple de la modalisation des compétences est le classement de S. Dylak (1995 : 38-39). En grandes lignes, l'auteur distingue trois niveaux de compétences, à savoir celui des compétences de base ; celui des compétences nécessaires et celui des compétences voulues. Les compétences de base permettent à l'enseignant de communiquer avec les apprenants et ses collègues de travail. Quant aux compétences nécessaires, elles se composent de trois types de sous-compétences, c'est-à-dire :

- les compétences interprétatives qui garantissent la congruence des conduites de l'enseignant dans toutes les circonstances ;

- les compétences autocréatives qui sous-tendent le développement de son savoir pédagogique ;

- les compétences de réalisation qui garantissent la mise en application des tâches éducatives.

Pour ce qui est des compétences voulues, elles restent en rapport avec les centres d'intérêts de l'enseignant et ses aptitudes, par exemple sportives, artistiques ou interpersonnelles, etc. Le mérite incontestable du classement susmentionné est qu'il permet d'opérationnaliser les compétences de l'enseignant : d'une part grâce à leur mise en structure selon les critères de valeur et d'utilité, d'autre part grâce au caractère suffisamment ouvert et abstrait des catégories de compétences distinguées. En fait, $\mathrm{S}$. Dylak fournit une matrice organisant et facilitant la réflexion sur les domaines des compétences de l'enseignant/de l'enseignant des LE.

Parmi les compétences qui marquent décidément leur présence dans les classements évoqués ci-dessus, se trouvent les compétences créatrices et innovantes. Ce qui témoigne de la reconnaissance de leur intérêt dans la formation des enseignants en Pologne, c'est qu'elles ont été considérées comme représentatives pour le métier de l'enseignant dans l'arrêté du Ministère de l'Éducation Nationale et des Sports du 
23.09.2003. Ainsi, suivant ce règlement officiel, durant leur formation les futurs enseignants devraient acquérir des compétences dans les domaines : didactique, éducatif et social, créatif, praxéologique, communicatif et information-média (www.lex.com. $\mathrm{pl} / \mathrm{du} / 2003 / 1655 . \mathrm{htm}$ ). D'après ce document officiel, les compétences créatrices se manifestent par :

- les capacités à s'autoformer et à innover,

- l'originalité des conduites,

- les aptitudes d'adaptation, de mobilité et de souplesse.

Cette entrée des compétences créatrices dans le répertoire des compétences de l'enseignant/l'enseignant des LE s'est faite en aval de l'intérêt pour le phénomène de la créativité dans différentes sphères de l'activité humaine. En fait, depuis la deuxième moitié du XX ${ }^{\text {ème }}$ siècle, le monde entre progressivement dans l'ère postmoderniste et postindustrielle qui est à la fois celle de homo creativus (Blachowska-Szmigiel, 2012). Les capacités créatrices et innovantes sont recherchées dans pratiquement tous les domaines de l'activité humaine, même les économistes parlent aujourd'hui de la gestion du savoir et revendiquent, dans ce contexte, la nécessité de gérer les talents. L'étendue de la popularité du phénomène de la créativité est due aux recherches des psychologues cognitifs et aux théories des psychologues humanistes. Selon les psychologues humanistes la créativité est une attitude, une façon d'être régulée par le besoin de réalisation (Kozielecki, 1995). Cette représentation de la créativité se manifeste dans la pédagogie de la créativité, laquelle prend pour objectif l'élaboration des théories pédagogiques du fonctionnement créatif de l'individu tout au long de sa vie ainsi que la préparation des programmes stimulant la créativité.

Quant aux psychologues cognitifs, ils démystifient le processus de la pensée créative et prouvent que la créativité est une propriété immanente de notre esprit. Une propriété qui se manifeste par les conduites heuristiques et non-conformistes sous-tendues par un ensemble de dispositions cognitives et de traits émotif-motivationnels dont le développement est stimulé ou freiné par l'environnement (Blachowska-Szmigiel, 2010 ; Pacteau, Lubart, 2011). Ceci conduit à l'élaboration de nombreuses techniques stimulant la pensée créative, appelée aussi la pensée synvergente (parmi lesquelles il convient d'indiquer la carte heuristique et le remue-méninges qui datent des années 60 . du $\mathrm{XX}^{\text {ème }}$ siècle) et des entraînements à la créativité (Nęcka, 2005).

Les dispositions cognitives et les traits conatifs créatifs ont donc une nature complexe et transversale. Néanmoins, compte tenu de nombreuses recherches effectuées à ce sujet depuis les années $60 \mathrm{du} \mathrm{XX} \mathrm{X}^{\text {ème }}$, parmi lesquelles il convient de mentionner celles de J.P. Guilford, E. de Bono, E. Nęcka, S. Popek, ou T. Lubart, il paraît que le noyau dur des dispositions et des traits créatifs de tout individu est constitué :

- au niveau cognitif par la pensée divergente soutenue dans le processus de la créativité par la pensée convergente, ce qui se traduit par un traitement de l'information dont le résultat est nouveau, original et de valeur ; 
- au niveau conatif par une bonne estime de soi. Ce trait de nature transversale relève d'une part des structures de la sphère émotive-motivationnelle, d'autre part il permet à l'individu de formuler des jugements sur le fonctionnement de son propre système cognitif. Une bonne estime de soi soutient le développement de l'individu. Débloquant ses structures cognitives et fortifiant sa motivation interne, il facilite son ouverture aux nouveautés, l'encourage à prendre des risques et à chercher des solutions innovatrices (Larivery, 2002 ; Blachowska-Szmigiel, 2010).

Le caractère complexe et transversal des dispositions et des traits créatifs suscite la question de l'optimisation de leur développement chez les futurs enseignants et les enseignants. La question que je me pose dans la suite de mes réflexions est donc comment accompagner les enseignants de FLE dans le développement de leur potentiel créatif de manière à le porter à son niveau de performance optimale.

\section{LE COACHING EN TANT QUE MÉTHODE D’ACCOMPAGNEMENT DE L'ENSEIGNANT DU FLE DANS LE DÉVELOPPEMENT DE SES COMPÉTENCES CRÉATIVES}

La formation institutionnelle des enseignants créatifs est réalisée en Pologne sous forme d'ateliers, d'entraînements, de cours, de spécialisations et d'études graduantes et postgraduantes. Parmi les centres universitaires qui les proposent se trouvent : Varsovie, Cracovie, Katowice, Lubin, Łódź, Gdańsk. Dans le cadre de « L’Éducation Permanente » il convient de mentionner les programmes tels que Leonardo da Vinci ou European Language Label. L'un met en valeur l'approche innovante de l'éducation et du développement professionnel, l'autre encourage les initiatives innovantes à tous les niveaux de l'enseignement/apprentissage des LE. Par ailleurs, la créativité est propagée aussi par des associations telles que Polskie Stowarzyszenie Kreatywności (l'Association Polonaise de la Créativité) (existant depuis l'an 2000) ou Polskie Stowarzyszenie Nauczycieli Twórczych (l'Association Polonaise des Enseignants Créatifs) (fondée en 2004). De son côté, le système de la promotion professionnelle oblige les enseignants à augmenter leurs compétences. Pour gravir les échelons de leur carrière professionnelle, les enseignants participent donc à différents stages ou études graduantes et postgraduantes. Par ailleurs, D. Ekierd-Oldroyd remarque à ce sujet que « la plupart des types de formations offertes est payante et c'est ainsi que l'enseignant fait gagner tout le monde bien qu'il y gagne peu luimême » (Ekiert-Oldroyd, 2003 : 129).

Sans contester la valeur et l'utilité de divers types de formations institutionnelles, il convient pourtant de remarquer que, de par leur nature, elles ne peuvent pas répondre aux besoins individuels des participants. Orientées vers l'ensemble du groupe, influencées par sa dynamique, elles suivent une progression standardisée. Cependant, vu le caractère complexe et transversal des dispositions et des traits créatifs, 
l'optimisation du niveau de leur performance demanderait que l'enseignant soit aussi accompagné et soutenu dans le processus de leur développement, aussi bien pendant ses études que durant sa carrière professionnelle. C'est bien dans ce contexte-là que s'inscrit la question des intérêts et des défis du coaching en tant qu'outil de libération du potentiel créatif des enseignants en FLE.

Le coaching fait partie d'une longue histoire d'accompagnement et son émergence s'inscrit «d'une part dans l'effondrement des institutions tutélaires telles que la famille, l'État, l'Église, l'armée, les syndicats, et d'autre part dans l'injonction faite à l'individu de devenir « lui-même » en dehors de ces institutions tutélaires et des constructions collectives - ce qui ne s'était encore jamais produit en histoire » (Halbout, 2009 : 5). Les origines du coaching remontent à l'antiquité, à la méthode maïeutique de Socrate. Cette méthode dont le nom vient de l'art de l'accouchement, consistait en une série de questions par lesquelles Socrate arrivait à faire découvrir à son interlocuteur son savoir implicite, intuitif. Elle permettait donc de libérer le potentiel de l'individu, et Socrate l'utilisait en alternance avec la méthode élenctique. L'objectif de cette dernière était de démontrer les faiblesses et les manques dans l'argumentation de son interlocuteur (Halbout, 2009).

En Europe, le mot « coach », dont le sens premier est « grande voiture à chevaux », commence à être utilisé au sens figuré d' " instructeur » vers 1830, et d' « entraîneur sportif » vers 1861. Au cours du $\mathrm{XX}^{\text {ème }}$ siècle l'usage du mot « coach » s'étend aux domaines de l'art, du sport et via ce dernier à la gestion. Bien que le coaching ne soit pas un phénomène nouveau et que ses racines remontent à l'antiquité, pour beaucoup son histoire commence aux États-Unis (Halbout, 2009 : 2). Prenant formes des entretiens, le coaching d'aujourd'hui a pour mission d'optimiser le développement des capacités et des ressources individuelles, d'équipe et d'entreprise. A l'heure actuelle, le coaching apparaît donc dans des contextes et des formes très diversifiés. Ainsi, le coach personnel aide à résoudre les problèmes, à développer le potentiel et l'estime de soi, le coach amoureux exerce le même rôle mais dans le domaine de la vie intime. Dans le contexte d'éducation, le coach commence à faire la concurrence (Bellego, 2009) au tuteur, et « le coaching pédagogique s'adresse aux élèves de l'école primaire ou secondaire, aux étudiants et à tout autre apprenant suivant des études choisies ou des formations imposées » (Mouzoune, $2010: 12$ ).

Comme une forme contemporaine d'accompagnement, le coaching fait couler beaucoup d'encre depuis déjà plus d'une dizaine d'années et suscite « de fortes réactions émotionnelles, de l'ordre de la répulsion, du doute, ou de l'enthousiasme inconditionnel » (Fatien, 2008 : 193). Quelles en sont les raisons ? D’abord, un flou qui entoure les pratiques du coaching renforce malheureusement la conviction que tout s'entraîne et que l'entraînement avec un coach suffit pour libérer ses ressources personnelles et remporter du succès. Ensuite, à la diversité des applications du coaching s'ajoutent les ambiguïtés dues au manque d'une définition représentative pour cette discipline. S. Persson-Gehin indique 53 définitions du coaching 
proposées entre 1992 et 2003 (Persson-Gehin [2005] In : Fatien 2008 : 194). Après, ce qui provoque un malaise par rapport au coaching en tant que discipline, c'est le caractère hétérogène de sa méthodologie. Issue de différents domaines des sciences humaines, tel que le management, la relation d'aide, la psychologie ou la communication, elle s'appuie sur des outils théoriques très variés : analyse transactionnelle, programmation neurolinguistique, process communication, théorie de l'intelligence émotionnelle, gestalt, approche systémique (Fatien, 2008). Enfin, le coaching en tant que métier peut soulever des questions de nature déontologiques quant à l'éthique du discours du coach, à la fiabilité de sa formation ou bien à ses dispositions professionnelles.

Tout comme un psychothérapeute, un journaliste ou bien un médecin, un coach suit un code éthique, ce qui n'empêche pourtant pas de se poser la question comment il réagirait dans le cas du conflit d'intérêt potentiel, par exemple lorsque les objectifs de la direction par laquelle il a été embauché, ne correspondent pas aux attentes des employés. Quant aux qualifications du coach, ce qui ressort d'un examen rapide des offres de la formation pour ce métier en Pologne, c'est que les cursus s'étalent sur en moyen 300 heures, réalisées en général sous forme d'ateliers :

- Akademia Set (l'Académie Set) (www.grupaset.pl) propose un cursus de 88 heures pour un coach et de 64 heures pour un coach systémique ;

- Grupa Trop (le Groupe Trop) (www.grupatrop.pl) présente un cursus divisé en deux modules dont le premier de base compte 137 heures et le suivant de spécialité est réalisé en 118 heures ;

- Instytut Rozwoju Biznesu (l'Institut du développement des Affaires) (www. irb.pl) offre environ 312 heures d'ateliers y compris les entretiens individuels.

Les cursus susmentionnés sont en principe destinés aux personnes désirant commencer leur aventure avec le coaching, aux étudiants, aux entraîneurs, aux directeurs des ressources humaines. Chacun peut s'y inscrire à condition d'en payer les coûts. Ce qui est commun pour ces cursus c'est qu'ils puisent dans le modèle américain et anglo-saxon du coaching. L'Institut du développement des Affaires se réfère à la tradition de l'école de Noble Manhattan Coaching, une tradition qui remonte à l'an 1992. Elle n'a donc que 20 ans environ. Quant à l'Académie Set, le programme de son cursus correspond aux exigences de International Coach Federation (la Fédération internationale des coachs) - organisation fondée en 1995. Nous apprenons également que son cursus se termine par un certificat « Associate Certified Coach ». Une autre approche du coaching est proposée Szkoła Wyższa Psychologii Społecznej (la Haute École de la Psychologie Sociale ; www.swps. $\mathrm{pl})$. Premièrement, elle demande aux candidats pour les études postgraduantes de coaching de passer par une interview de recrutement. De plus, avant de passer au bloc de coaching (98 heures), les étudiants réalisent un bloc psychologique consacré aux propriétés universelles du fonctionnement de l'homme ( 80 heures). Sans aucun doute, le souci de sélectionner les candidats et ensuite de donner aux étudiants des 
bases psychologiques sont des avantages des études proposées par la Haute École de la Psychologie Sociale. Une fois le contexte psychologique explicité, la méthodologie du coaching gagne de l'envergure.

Ce dont il faut absolument se méfier c'est l'illusion que chacun peut devenir coach. Un coach doit savoir pousser son client à une autoréflexion, à développer ses capacités métacognitives et à l'amener progressivement à des prises de conscience de son potentiel. Son rôle fait penser à celui du chapeau bleu de la méthode des six chapeaux de la réflexion de E.de Bono (2011). Comme le souligne R.-M. Halbout « Idéalement le coach ne vise rien pour celui qu'il accompagne. Il est juste en mesure de créer un espace d'introspection, de réflexion et donc de transformation » (Halbout, 2009:2-3). Un coach devrait donc travailler sur lui-même, se questionner, savoir se remettre en cause et en même temps se distinguer par une stabilité personnelle (Halbout, 2009 : 2-3). Malheureusement la popularité croissante du coaching en Pologne renforce la conviction qu'il suffit de suivre une formation afin d'exercer ce métier. On le voit à l'exemple du projet réalisé dans 20 écoles de la Grande Pologne en 2013 (IDMNI, 2013) où le rôle de coach est attribué au directeur. Financé par l'Union Européenne, ce projet a duré 6 mois et a été réalisé dans le groupe de 108 personnes (dont 22 hommes et 86 femmes). Son objectif était de fournir un outil de motivation pour l'innovation. La tâche du directeur-coach consistait entre autres à éveiller la motivation interne des enseignants, à renforcer leur engagement et à travailler sur les effets de leur travail. Pour remplir le rôle de coach, les directeurs ont suivi une formation. Pourtant dans les caractéristiques du projet le lecteur ne trouve d'informations ni sur la durée, ni sur le nombre d'heures. Ainsi, malgré les commentaires et les opinions favorables voire enthousiastes que nous lisons dans la description du projet, celui-ci suscite une certaine défiance et éveille des questions, entre autres sur les dispositions personnelles des directeurs pour exercer le métier de coach. Au lieu d'attribuer au directeur le rôle de coach, il serait peut-être plus juste de parler dans le cadre dudit projet des techniques de coaching mises au service de la motivation.

Il semble que le coaching ne soit pas une mode passagère mais une tendance forte, témoignant de l'intérêt croissant pour l'individu. La libération de son potentiel est repensée dans le contexte de la libération du potentiel du groupe, de l'organisation, voire de la société. Ainsi, nous y voyons émerger l'approche systémique de l'être humain. Tout en restant un être intradirigé et autonome il est considéré comme un constituant indispensable du groupe, de l'organisation, de la société, etc. Au lieu donc de combattre en vain le coaching, il faudrait se poser la question quel coach et quel coaching proposer pour libérer le potentiel créatif de l'enseignant de FLE. Avant tout un coaching qui relève de la tradition philosophique et qui s'inscrit dans la tradition d'accompagnement. L'avantage de cette approche présentée par R.-M. Halbout (2009) est qu'elle préserve nos racines culturelles en montrant une ouverture vers les horizons nouveaux. Quant au coach, pour mieux cerner la problématique individuelle du coaché et mieux adapter les outils à ses besoins, il devrait également 
avoir une formation psychologique. Pour les futurs enseignants, le coaching devrait s'inscrire dans le cadre de leurs études et constituer un prolongement des cours et des ateliers de la créativité. Pour les enseignants exerçant leur métier, il serait un complément des stages ou des ateliers. Les séances de coaching devraient permettre aux enseignants de :

- réviser les savoirs et les savoir-faire acquis pendant les cours, les ateliers ou les stages,

- envisager ces savoir et savoir-faire dans une perspective individuelle,

- prendre conscience des possibilités de la réutilisation de ces savoirs et savoirfaire en classe de FLE,

- développer la réflexion sur leur fonctionnement cognitif, leur style d'apprentissage et d'enseignement, ainsi que sur leurs propres limites ou obstacles aux comportements créatifs,

- prendre du recul par rapport à eux-mêmes,

- franchir les obstacles de leurs comportements créatifs, bien entendu s'ils ne sont pas dus aux blocages intrapsychiques profonds au point de demander l'aide d'un psychothérapeute,

- prendre conscience de leurs besoins et attentes,

- planifier leur développement professionnel.

\section{CONCLUSION}

Après avoir terminé ses études, l'enseignant de FLE en Pologne approfondit ses compétences professionnelles en participant aux diverses formations. Il le fait non seulement pour construire son autorité et pour être à même de jouer le rôle de guide du sujet apprenant mais aussi parce qu'il est pris au piège du système de promotion. Au centre de son intérêt se trouvent entre autres les compétences créatives. L'objectif de ma réflexion était d'examiner les intérêts et les défis du coaching en tant que méthode d'accompagnement de l'enseignant de FLE dans le développement de ses compétences créatives. Pour libérer le potentiel créatif et pour optimiser son développement, il serait idéal que l'enseignant complète les ateliers, les cours ou les stages de créativité par les séances avec un coach, à condition pourtant que ce coaching ne soit pas copié prétentieusement sur le modèle américain mais qu'il relève de la tradition philosophique et qu'il s'inscrive dans le contexte psychologique. En d'autres termes, le coaching " européen » devrait être pensé dans le cadre des études psychologiques comme une des spécialités possibles. 


\section{RÉFÉRENCES}

BeLlego, Jean-Yves (2009): Le coaching pédagogique. Apprendre à apprendre. Le céfs de la réussite. Paris : Ellaboré Éditions.

Bono de, Edward (2011): Les six chapeaux de la réflexion. Paris : Éditions Eyrolles.

Bilińska-SuchaneK, Ewa; JARECKA-ŻYluK, Małgorzata (2005): „Przygotowanie nauczyciela do dekonstrukcji kultury w edukacji. Model kształtowania postawy krytycznej ucznia”. In : Wolfgang Hörner, Mirosław S. SzYMańSki (éd.), Nauczyciel i kształcenie nauczycieli. Zmiany i wyzwania, Warszawa: Wydawnictwo Akademickie Żak, 46-69.

BlachowSKa-Szmigiel, Marzena (2010): Twórcze schematy poznawcze a kreatywność językowa. Na przykładzie języka francuskiego jako obcego. Poznań : Wydawnictwo Naukowe UAM.

BlachowsKa-Szmigiel, Marzena (2013): „Zadania zorientowane na ekspresję teatralną jako narzędzie wspierające rozwój kreatywności językowej studenta filologii romańskiej” In : Elżbieta Nowikiewicz (éd.), Kompetencje ogólne. Kształcenie filologów. Neofilolog nr 40/1, Poznań: PTN, 29-41.

BogdAŃSKI, Marek (2001): „Samodzielność ucznia jako cel w zreformowanej szkole” In : Edukacja jutra V Tatrzańskie Seminarium Naukowe. Częstochowa : Agencja Promocji Nauki i Kultury MENOS s.c., 145-151.

Conseil De La Coopération Culturelle Comité de l'Éducation « Apprentissage des langues et citoyenneté européenne » (2001): Un cadre européen commun de référence pour les langues : apprendre, enseigner, évaluer. Paris : Didier.

Dylak, Stanisław (1995): Wizualizacja w kształceniu nauczycieli, Poznań : Wydawnictwo Naukowe UAM.

EKIERT-OldROYD, Dorota (2003): „Pedeutologiczne konteksty dydaktyki twórczości i ich pragmatyczne Implikacje. Pedeutologia twórczości a dydaktyka twórczości”. In : Krzysztof J. SzmIDT (éd.), Dydaktyka twórczości. Kraków : Impuls, 135-158.

Halbout, Reine-Marie (2009): Savoir être coach : un art, une posture, une éthique. Paris : Éditions Eyrolles.

JARoszewSKA, Anna (2009): „Analiza kompetencji nauczycieli języków obcych w kontekście nauczania w różnych grupach wiekowych" In : Mirosław Pawlak, Anna Mystkowska-Wiertelak, Agnieszka Pietrzykowska (éd.), Nauczyciel języków obcych dziś i jutro. Poznań - Kalisz : Wydział Pedagogiczno-Artystyczny UAM w Kaliszu, Uniwersytet im. Adama Mickiewicza w Poznaniu, 83-94.

KozIELECKI, Józef (1995): Koncepcje psychologiczne człowieka, Warszawa : Wydawnictwo „Żak”.

KwIECIŃSKI, Zbigniew (2000): Tropy - ślady - próby: studia i szkice z pedagogiki pogranicza. Poznań : Wydawnictwo Edytor.

LASKA, Eugenia Iwona (2007): „W poszukiwaniu wyznaczników edukacyjnej aktywności nauczyciela. Samokształcenie”, In : Eugenia Iwona Laska (éd.), Nauczyciel między tradycją a wspótczesnościa. Teoria i praktyka. Rzeszów : Wydawnictwo Uniwersytetu Rzeszowskiego, 108-115.

Martinez, P. (1996): La didactique des langues étrangères. Paris : PUF. Collection « Que sais-je? ».

Mouzoune, Karim (2010): Le coaching pédagogique. Comment vaincre les difficultés scolaires. Paris : L'Harmatton.

NĘCKA, Edward (2005): Psychologia twórczości. Gdańsk : Gdańskie Wydawnictwo Psychologiczne.

OŚRodek RozwoJu EduKacJI (2013): Instrukcja do dwuczynnikowego modelu motywowania nauczycieli do innowacyjności. Poznań.

Senge, Peter (1998): Piąta dyscyplina. Teoria i praktyka organizacji uczacych się. Warszawa : Dom Wydawniczy ABC.

StrYкоwSKI, Wacław (2005): „Kompetencje współczesnego nauczyciela”, Neodidagmata 27/28 : $15-28$ 
TARaszkiewicz, Małgorzata (2001): Jak uczyć jeszcze lepiej! Szkoła petna ludzi. Poznań : Wydawnictwo Arka.

WiLCZYŃSKA, Weronika (1999): Uczyć się czy być nauczanym? O autonomii w przyswajaniu języka obcego. Warszawa-Poznań: Wydawnictwo Naukowe PWN SA.

ZAWADZKA, Elżbieta (2004): Nauczyciele języków obcych w dobie przemian. Wydawnictwo: Oficyna Wydawnicza Impuls.

ŻuKowsKa Zofia (1993): „Nauczyciel: człowiek - pedagog - specjalista”. Wychowanie Fizyczne i Zdrowotne, $\mathrm{nr}$ 4: 7-12.

SOURCES ÉLECTRONIQUES

Source électronique 1 :

FAtIEN, Pauline (2008): «Des ambiguïtés des mots/maux de coaching » In : Eres. Nouvelle revue de psychosociologie.2008/2 - $n^{\circ}$ 6. <http://www.cairn.info/revue-nouvelle-revue-de-psychosociologie-2008-2-page-193.htm>, 193-211. Consulté le 10 mars 2014.

Source électronique 2 :

DeREŃ, Edward (2011) : Nauczyciel - zawód czy misja i powołanie? < https://sites.google.com/site/ ksztalcenienauczycieli/nauczyciel---zawod-czy-misja-i-powolanie>. Consulté le 5 janvier 2014.

Source électronique 3 :

MeIRIEu Philippe (2001): L'éducation et le rôle des enseignants à l'horizon 2020. Paris : UNESCO. $<$ http://www.meirieu.com/ ... TIONNELS/UNESCO2020.pdf>.

Source électronique 4 :

Pacteau Chantal, LuBART, Todd (2011) : Le développement de la créativité <http://www.scienceshumaines.com/le-developpement-de-la-creativite_fr_5213.html>. Consulté le 2 janvier 2013.

Source électronique 5 :

Larivery, Michelle (2002): «L'estime de soi » In : La lettre du psy. Volume $6 \mathrm{n}^{\circ} 3 \mathrm{c}$ mars $<\mathrm{http} / / /$ www.redpsy.com/infopsy/estime.html>. Consulté le 18 mars 2014.

Source électronique 5 :

$<$ www.lex.com.pl/du/2003/1655.htm>. Consulté le 5 janvier 2010.

Source électronique 6 :

<www.urb.pl>. Consulté le 10 avril 2014.

Source électronique 7 :

<www.grupatrop.pl>. Consulté le 10 avril 2014.

Source électronique 8:

<www.grupaset.pl>. Consulté le 10 avril 2014.

Source électronique 9 :

<www.swps.pl>. Consulté le 10 avril 2014. 